\title{
Alterations in the Gut Microbiota and Hepatitis-B-Virus Infection in Southern Chinese Patients With Coexisting Non-Alcoholic Fatty Liver Disease and Type-2 Diabetes Mellitus
}

\author{
Weijia Han ${ }^{1,2}$, Chunyang Huang ${ }^{3}$, Yali Ji ${ }^{2}$, Ling Zhou ${ }^{2}$, Jinjun Chen ${ }^{2,45 *}$ and Jinlin Hou ${ }^{1 *}$ \\ ${ }^{1}$ Department of Liver Disease Center, Shenzhen Hospital, Southern Medical University, Shenzhen, China, ${ }^{2}$ Hepatology Unit, \\ Department of Infectious Diseases, Nanfang Hospital, Southern Medical University, Guangzhou, China, ${ }^{3}$ Second Department \\ of Liver Disease Center, Beijing Youan Hospital, Capital Medical University, Beijing, China, ${ }^{4}$ Hepatology Unit, Zengcheng \\ Branch, Nanfang Hospital, Southern Medical University, Guangzhou, China, ${ }^{5}$ Chinese (Acute on) Chronic Liver Failure \\ Consortium (Ch-CLIF.C), Shanghai, China
}

Background: Hepatitis B virus (HBV) infection has been reported to affect the bacterial characteristics in the host. We aimed to elucidate the compositional and functional

OPEN ACCESS

Edited by:

Jinhang Gao,

Sichuan University, China

Reviewed by:

Hao Wu,

Sichuan University, China

Giovanni Tarantino,

University of Naples Federico II, Italy Jia Yao,

Shanxi Medical College, China

*Correspondence:

Jinjun Chen

chij@smu.edu.cn

Jinlin Hou

jlhousmu@163.com

Specialty section

This article was submitted to

Gastroenterology,

a section of the journal

Frontiers in Medicine

Received: 29 October 2021 Accepted: 23 November 2021 Published: 21 December 2021

Citation:

Han W, Huang C, Ji Y, Zhou L, Chen J

and Hou $\mathrm{J}$ (2021) Alterations in the Gut Microbiota and Hepatitis-B-Virus Infection in Southern Chinese Patients

With Coexisting Non-Alcoholic Fatty

Liver Disease and Type-2 Diabetes Mellitus. Front. Med. 8:805029.

doi: 10.3389/fmed.2021.805029 characteristics of the microbiota in southern Chinese patients with coexistent HBV infection, non-alcoholic fatty liver disease (NAFLD), and type-2 diabetes mellitus (T2DM).

Methods: Healthy controls (HCs) and patients with coexistent NAFLD and T2DM were enrolled. Patients were divided into two groups: N1 (without HBV infection) and N2 (with HBV infection). Stool samples were collected for 16s RNA gene sequencing and untargeted metabolomics analysis.

Results: Bacterial diversity was decreased in the N2 group. There was a significantly lower abundance of bacteria of Faecalibacterium, Gemmiger, and Clostridium_XIVA genera, but a higher abundance of Megamonas and Phascolarctobacterium genera in the N2 group. Compared with the N1 group, the abundance of Gemmiger species was even lower, and alterations in the abundance of Phascolarctobacterium and Clostridium_XIVA genera only occurred in the N2 group. There were significantly different fecal metabolic features, which were enriched in glucose and lipid metabolic pathways (e.g., fatty acid and glycerophospholipid metabolism) between the N2 and HC groups. Metabolites in glycerophospholipid metabolism, such as Sn-3-o-(geranylgeranyl)glycerol1-phosphate, were even higher in the N2 group than in the N1 group. The decreased Faecalibacterium and Gemmiger contributed to the increased level of Sn-3-o-(geranylgeranyl) glycerol1-phosphate, palmitoylcarnitine, and serum triglycerides. Clostridium_XIVA species were positively correlated to 15(s)-hpete. Megamonas species were positively correlated with the serum level of glucose indirectly.

Conclusions: The distinct gut-microbiome profile associated with HBV infection has a role in lipid metabolism and glucose metabolism in patients with coexistent NAFLD and T2DM.

Clinical Trial Registration: www.ClinicalTrials.gov, identifier: NCT03525769.

Keywords: HBV, NAFLD, microbiota, metabolome, hepatitis B virus 


\section{INTRODUCTION}

Non-alcoholic fatty liver disease (NAFLD) is defined by fat accumulation in the liver. It is an important health issue worldwide. Gradually, NAFLD is becoming the most common cause of further progression to end-stage liver diseases (1). The increasing prevalence of NAFLD is related to type-2 diabetes mellitus (T2DM). Approximately 55\% of patients with NAFLD also have T2DM (2). Patients with coexisting NAFLD and T2DM are 2-4-times more likely to develop end-stage liver diseases than those without T2DM (3). Excess glucose is the raw material for the synthesis of free fatty acids, which leads to increased levels of cholesterol and triglycerides (TG). Lipid accumulation due to increased glucose levels can cause liver inflammation in NAFLD $(4,5)$.

The term "microbiota" is a collective term for the microorganisms that live in or on the human body. "Microbiome" refers to the collection of genomes from all the microorganisms in an environment (e.g., gut). Microbiota dysbiosis is related to an increased serum level of TG and glucose tolerance in NAFLD and T2DM $(6,7)$. Several studies have summarized the altered composition of the microbiome in NAFLD (8), T2DM (9), and coexistence of NAFLD and T2DM (10). Foods (11) or drugs (12) that improve microbiota dysbiosis and alter microbiome composition may reduce plasma levels of glucose and TG in patients with NAFLD or T2DM.

The number of patients who have hepatitis $B$ virus (HBV) infection, NAFLD, and T2DM is high (13). The prognosis of coexisting chronic hepatitis $\mathrm{B}(\mathrm{CHB})$ and NAFLD is worse than that of CHB alone or NAFLD alone $(14,15)$. Moreover, the HBV has been reported to affect bacterial characteristics in the host $(16,17)$. Hence, the compositional and functional characteristics of the microbiota in patients with coexisting $\mathrm{HBV}$ infection, NAFLD, and T2D merit exploration.

We recruited healthy people and patients with coexisting NAFLD and T2DM. The bacterial composition and functional characteristics of patients were analyzed through 16s RNA gene sequencing and untargeted metabolomics analysis. Our study provides ideas for future longitudinal studies to investigate the effect of the microbiota in the treatment of patients with coexistent HBV infection, NAFLD, and T2DM.

\section{METHODS}

\section{Ethical Approval of the Study Protocol}

The study protocol was approved (NFEC-2018-023) by the Ethics Committee of Nanfang Hospital within Southern Medical University (Guangzhou, China) and registered at ClinicalTrials.gov (NCT04573283). All participants provided written informed consent for their data to be used in this study.

\section{Inclusion Criteria}

The inclusion criteria for healthy controls (HCs) and patients were: (1) age $\geq 18$ years; (2) men and women; (3) microecological agents (MAs) and antibiotics were not used; (4) excess alcohol was not consumed (alcohol intake $<140 \mathrm{~g} /$ week for men; $<70$ $\mathrm{g} /$ week for women).
In addition to the four criteria mentioned above, two additional criteria were applicable for patients with coexisting NAFLD and T2DM (N1 group). The first criterion was that NAFLD was detected by transient elastography using FibroScan $^{\mathrm{TM}}$ (Echosens, Paris, France). Liver biopsy is not acceptable for long-term dynamic monitoring of NAFLD stages. Therefore, non-invasive methods for NAFLD assessment have become essential. The controlled attenuation parameter (CAP) has demonstrated good accuracy for reflecting NAFLD severity $(18,19)$. In a recent study on gut microbiome (GM) signatures and the biological functions associated with liver fibrosis, Kwan et al. (20) used FibroScan to screen for NAFLD. In the present study, CAP > $236(\mathrm{~dB} / \mathrm{m})$ was diagnostic for NAFLD. The second criterion was that the T2DM diagnosis was assisted by the Endocrinology Department of Nanfang Hospital.

In addition to the criteria mentioned above, patients with coexisting HBV infection, NAFLD, and T2DM (N2 group) also had to meet one additional criteria: hepatitis B surface antigen (HBsAg) positivity with detectable HBV-DNA in serum, according to PCR.

\section{Exclusion Criteria}

The exclusion criteria were people: (i) with symptoms of acute or chronic infection; (ii) with cancer; (iii) who were pregnant or lactating; (iv) using antibiotics or intestinal MAs within 2 weeks of enrollment.

\section{Patients}

This prospective observational study was carried out between October 2020 and December 2020 at Nanfang Hospital. HCs and patients with coexistent NAFLD and T2DM were enrolled. Patients underwent standard treatment for their particular disease regardless of their enrollment in this study (Figure 1).

Enrolled patients underwent sampling of stools and blood simultaneously. Levels of alanine aminotransferase (ALT), aspartate transaminase (AST), total bilirubin (TBIL), triglycerides (TG), total cholesterol (TC), and glucose were included as biochemical parameters in serum. Fecal samples were obtained in a plastic collection kit. All samples were stored at $-80^{\circ} \mathrm{C}$.

\section{6s RNA Gene Sequencing in Stools}

The DNA in the microbial community was extracted using the MagPure Stool DNA KF Kit B (Magen, Beijing, China) according to manufacturer instructions. DNA was quantified

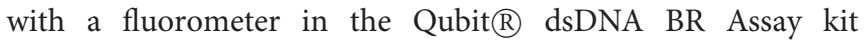
(Invitrogen, Carlsbad, CA, USA). DNA quality was checked by running aliquots on $1 \%$ agarose gel.

Variable region (V4) of the bacterial $16 \mathrm{~S}$ rRNA gene was amplified with the degenerate PCR primers 515 forward $\left(5^{\prime}\right.$-GTGCCAGCMGCCGCGGTAA-3 $\left.{ }^{\prime}\right)$ and 806 reverse $\left(5^{\prime}\right.$ GGACTACHVGGGTWTCTAAT-3'). Forward and reverse primers were tagged with an adapter, pad, and linker sequences from Illumina (San Diego, CA, USA). PCR enrichment was done in a reaction volume of $50 \mu \mathrm{l}$ containing $30 \mathrm{ng}$ of template, fusion PCR primer, and PCR master mix. PCR cycling conditions were $95^{\circ} \mathrm{C}$ for $3 \mathrm{~min}, 30$ cycles of $95^{\circ} \mathrm{C}$ for $45 \mathrm{~s}, 56^{\circ} \mathrm{C}$ for $45 \mathrm{~s}$, 


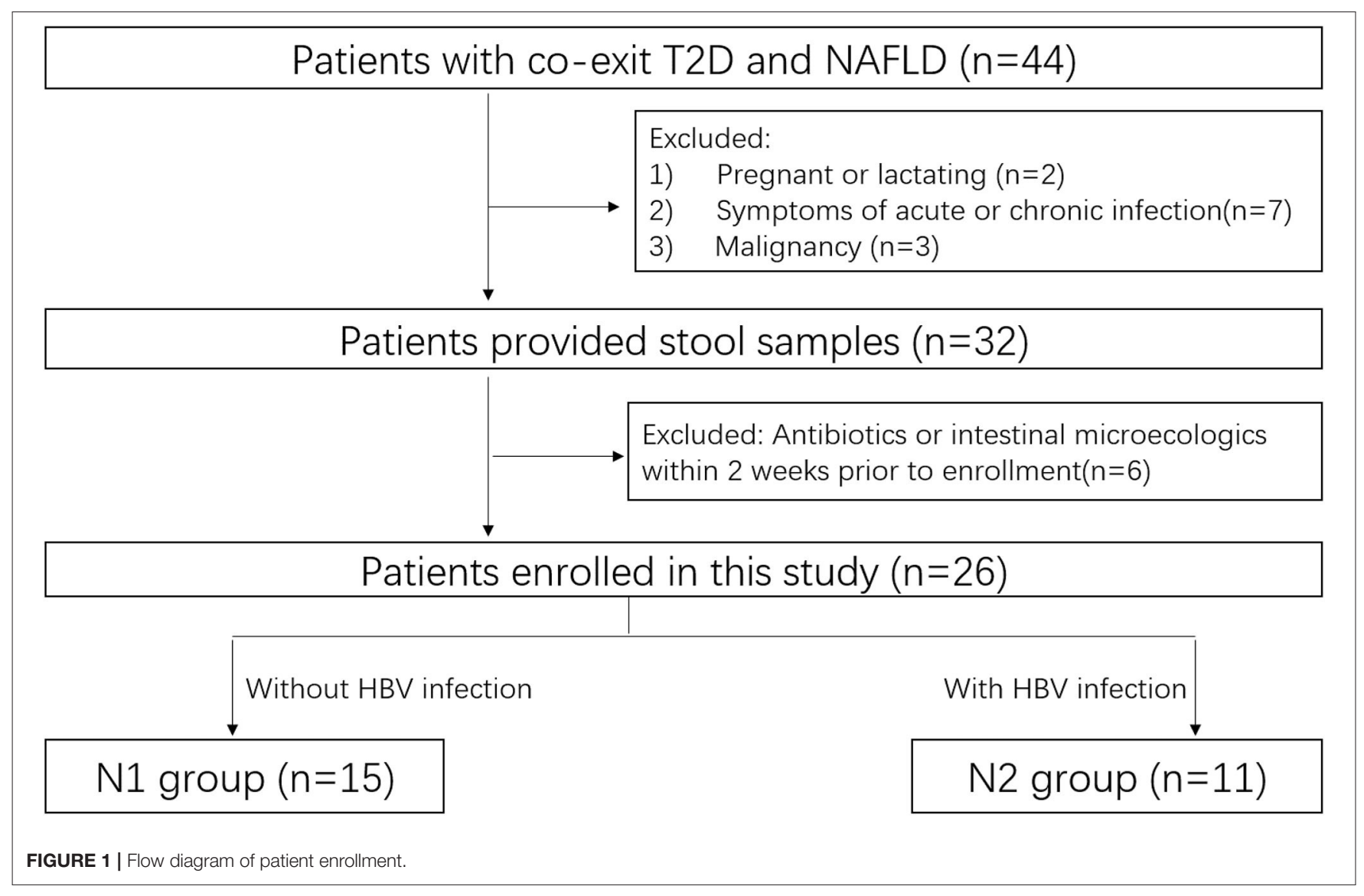

$72^{\circ} \mathrm{C}$ for $45 \mathrm{~s}$, and final extension at $72^{\circ} \mathrm{C}$ for $10 \mathrm{~min}$. PCR products were purified using Agencourt AMPure XP beads and eluted in elution buffer (Beckman Coulter, Fullerton, CA, USA). Libraries were qualified by a bioanalyzer (2,100 series; Agilent Technologies, Santa Clara, CA, USA). Validated libraries were used for sequencing on the HiSeq 2,500 platform, following the standard pipelines of Illumina, and generated $2 \times 250 \mathrm{bp}$ paired-end reads.

\section{Untargeted Metabolomics Analysis Using Stools}

Stool samples were used for untargeted metabolomics analysis. A sample $(100 \mathrm{mg})$ was weighed precisely and mixed with 800 $\mu l$ of extract (methanol:acetonitrile:water $=2: 2: 1 \quad(v: \quad v: v)$, precooled at $-20^{\circ} \mathrm{C}$ ). Then, the sample was homogenized at $50 \mathrm{~Hz}$ for $5 \mathrm{~min}$ by a tissue lyser with two glass beads. Then, $10 \mathrm{~min}$ of ultrasound agitation in water at $4^{\circ} \mathrm{C}$ and for $1 \mathrm{~h}$ at $-20^{\circ} \mathrm{C}$ was undertaken. After centrifugation at 25,000 rpm for $15 \mathrm{~min}$ at room temperature, the sample was mixed with $600 \mu \mathrm{l}$ of complexation solution (methanol: $\mathrm{H}_{2} \mathrm{O}=1: 9(v: v)$ ), followed by vortex-mixing for $1 \mathrm{~min}$, ultrasound agitation in water for $10 \mathrm{~min}$ at $4^{\circ} \mathrm{C}$, and centrifugation at $25,000 \mathrm{rpm}$ for $15 \mathrm{~min}$ at $4^{\circ} \mathrm{C}$. Then, two-dimensional ultrahigh pressure liquid chromatography was done using a Waters system (Waltham, MA, USA). Tandem mass spectrometry was carried out using a $\mathrm{Q}$ Exactive ${ }^{\mathrm{TM}}$ high-resolution mass spectrometer (Thermo Fisher
Scientific, Waltham, MA, USA) for the separation and detection of metabolites.

\section{Bioinformatics Analysis}

Filtering and remaining high-quality clean data for analysis. Reads were spliced into tags. Selection of operational taxonomic units (OTUs) was achieved using a similarity cutoff of $97 \%$. We clustered Tags into OTUs through USEARCH 7.0.1090. Species annotation was achieved by comparing OTUs with the Ribosomal Database Project (release date $=3$ September 2021). Based on OTUs and annotation results, $\alpha$-diversity and $\beta$-diversity were displayed. The Wilcoxon test was used to calculate $\alpha$-diversity. The Wilcoxon rank-sum test was used to calculate $\beta$-diversity. The distinguished genus and predicted pathway were screened based on the Wilcoxon test $(p<0.05)$ and $\mid \log$ base 2 -fold change (FC) $\mid>1$ through PICRUSt2 v2.2.0b, R (v3.4.10). Linear discriminant analysis effect size (LEfSe) (https://huttenhower.sph.harvard.edu/galaxy/) was employed to explore different microbiota at different taxonomic levels (LEfSe is a software tool for finding higher-dimensional biomarkers and revealing genomic characteristics to assess whether differential microbiotas are expected.). The Spearman correlation coefficient revealed important patterns and relationships among dominant species. GMrepo (https://gmrepo.humangut.info/home) (8) was used to search the differential gut microbiota. At the class level, the microbiota with relative abundance $<0.5 \%$ in all 
TABLE 1 | The baseline characteristic of N1 and N2 group.

\begin{tabular}{|c|c|c|c|c|c|}
\hline & Health (CON) & $\begin{array}{l}\text { NAFLD and } \\
\text { T2D (N1) }\end{array}$ & $\begin{array}{c}\text { CHB } \\
\text { accompanied by } \\
\text { NAFLD and T2D } \\
\text { (N2) }\end{array}$ & $P^{*}$ & $\mathbf{P}^{\star *}$ \\
\hline $\mathrm{n}$ (male) & $16(10)$ & $15(6)$ & $11(2)$ & & \\
\hline Age (year) & $53.8 \pm 9.84$ & $47.67 \pm 9.74$ & $52.73 \pm 6.6$ & 0.150 & 0.75 \\
\hline Height (cm) & $165.93 \pm 8.81$ & $162.87 \pm 8.75$ & $162.95 \pm 8.25$ & 0.980 & 0.39 \\
\hline Weight (kg) & $62.2 \pm 8.79$ & $72.88 \pm 11.45$ & $68.15 \pm 10.95$ & 0.299 & 0.13 \\
\hline $\mathrm{BMI}$ & $22.48 \pm 1.52$ & $27.48 \pm 3.59$ & $25.53 \pm 2.54$ & 0.138 & 0.00 \\
\hline Waistline (cm) & & $94.2 \pm 8.59$ & $91.55 \pm 5.75$ & 0.384 & \\
\hline Heart rate & & $74.67 \pm 9.71$ & $81.1 \pm 12.84$ & 0.167 & \\
\hline ALT (IU/L) & & $41.67 \pm 33.95$ & $36.52 \pm 19.88$ & 0.658 & \\
\hline AST (IU/L) & & $24.2 \pm 12.83$ & $27.97 \pm 15.41$ & 0.503 & \\
\hline TBIL ( $\mu \mathrm{mol} / \mathrm{L})$ & & $28.95 \pm 52.76$ & $20.81 \pm 19.06$ & 0.631 & \\
\hline $\mathrm{TG}(\mathrm{mmol} / \mathrm{L})$ & & $1.88 \pm 1.22$ & $2.45 \pm 1.62$ & 0.315 & \\
\hline TC (mmol/L) & & $5.44 \pm 1.32$ & $5.25 \pm 1.11$ & 0.704 & \\
\hline GLU (mmol/L) & & $9.11 \pm 4.52$ & $6.57 \pm 1.09$ & 0.082 & \\
\hline $\mathrm{CAP}(\mathrm{dB} / \mathrm{m})$ & $<236$ & $\begin{array}{c}315.23 \pm \\
38.59\end{array}$ & $286.6 \pm 36.53$ & 0.068 & \\
\hline
\end{tabular}

*The comparison between N1 and N2 group; ${ }^{*}$ The comparison between N2 and CON group.

ALT, Alanine transaminase; AST, Aspartate transaminase; TBIL, Total bilirubin; TG, Triglyceride; TC, Total cholesterol; GLU, Glucose. The meaning of the bold values: $p<$ 0.05 .

samples were merged into "Others." The metabolome database of the Beijing Genomics Institute was used to analyze the key primary metabolites and intermediates of key metabolic pathways. The Human Metabolome Database (https://hmdb. $\mathrm{ca} /$ ) was used to analyze human metabolites. The Lipidmaps (www.lipidmaps.org/resources/databases/index.php/) database was employed to identify the metabolites of lipids. Partial least squares-discriminant analysis (PLS-DA) was employed to analyze differences in metabolites between groups. The Kyoto Encyclopedia of Genes and Genomes (KEGG) database (www.genome.jp/) was used for the analysis of enrichment of the metabolic pathways of different metabolites.

\section{Statistical Analyses}

Continuous variables are expressed as the mean \pm SD. A $p<0.05$ was considered significant. Statistical analyses were undertaken using SPSS 19 (IBM, Armonk, NY, USA). $\alpha$-Diversity was evaluated using mothur-1.39.5/mothur and ggplot packages, and $\beta$-diversity was evaluated using QIIME1 and ggplot packages, within R 3.2.1 (R Institute for Statistical Computing, Vienna, Austria). Principal coordinates analysis (PCoA) was done using the mixOmics package within $\mathrm{R}$.

\section{RESULTS}

\section{Characteristics of the Entire Cohort}

The clinical characteristics of the entire cohort are shown in Table 1. There were no significant differences in age, height, weight, waist circumference, or heart rate between HCs and the
$\mathrm{N} 2$ group $(p>0.05)$. However, the body mass index (BMI) of HCs $\left(22.48 \pm 1.52 \mathrm{~kg} / \mathrm{m}^{2}\right)$ was significantly lower than that of the $\mathrm{N} 2$ group $\left(25.53 \pm 2.54 \mathrm{~kg} / \mathrm{m}^{2}\right)(p<0.05)$. There were no significant differences in age, height, weight, waist circumference, heart rate, BMI, CAP, or levels of ALT, AST, TBIL, TG, TC, or glucose between the N1 and N2 groups $(p>0.05)$.

\section{Bacterial Characteristics of Patients With Coexistent HBV Infection, NAFLD, and T2DM}

The saturated rarefaction curves based on the Shannon index and observed species indicated the high quality of sequences for all samples (Supplementary Figure 1). The richness and diversity of the $\mathrm{HC}$ and $\mathrm{N} 2$ groups were compared through the $\alpha$-diversity metrics shown in Figure 1. There was no significant difference in the Chao index, Simpson index, or Ace index $(p>0.05)$. The coverage index of the N2 group was significantly higher than that of the HCs group $(p<0.05)$, whereas the Sobs index and the Shannon index of the N2 group were significantly lower than those of the HCs group $(p<0.05)$ (Figure 2A). The $\beta$-diversity of the $\mathrm{N} 2$ group was significantly lower than that of the HCs group $(p<0.05)$ (Figure 2B). The $\beta$-diversity heatmap showed higher indices between groups (Figure 2C). Significant differences in bacterial communities were analyzed through PCoA. The microbiota were separated in the first axis (PC1) (Supplementary Figure 2).

The bacterial composition of the N2 group was explored (Figure 2). At the phylum level, LEfSe clustering showed that microbiota of the HC group were concentrated in Firmicutes and Candidatus_Saccharibacteria, whereas those of the N2 group were concentrated in Bacteroidetes and Fusobacteria (Figures 3A,B). The Wilcoxon test showed that the abundance of Firmicutes was significantly higher in the HC group, whereas the abundance of Bacteroidetes and Fusobacteria was significantly higher in the N2 group $(p<0.05)$ (Figure 3C). At the order level, the HC group was concentrated in Clostridiales, Caulobacterales, and Erysipelotrichales, and the N2 group was concentrated in Bacteroidales and Fusobacteriales (Figures 3A,B). At the genus level, there were 21 differentially expressed microbiota between the two groups (Supplementary Table 1). The Wilcoxon test showed that the abundance of Faecalibacterium, Gemmiger, and Clostridium_XIVA species was decreased significantly, whereas the abundance of Megamonas and Phascolarctobacterium species was increased significantly in the N2 group $(p<0.05)$ (Figure 3D).

These data indicated a significant alteration of microbiota composition in patients with coexistent HBV infection, NAFLD, and T2DM. Faecalibacterium, Gemmiger, Clostridium_XIVA, Megamonas, and Phascolarctobacterium were the distinct genera in the GM.

\section{HBV Infection Affects the Bacterial Characteristics of Patients With Coexistent NAFLD and T2DM}

$\alpha$-Diversity and $\beta$-diversity do not show a significant difference between the N1 group and N2 group $(p>0.05)$ 
A
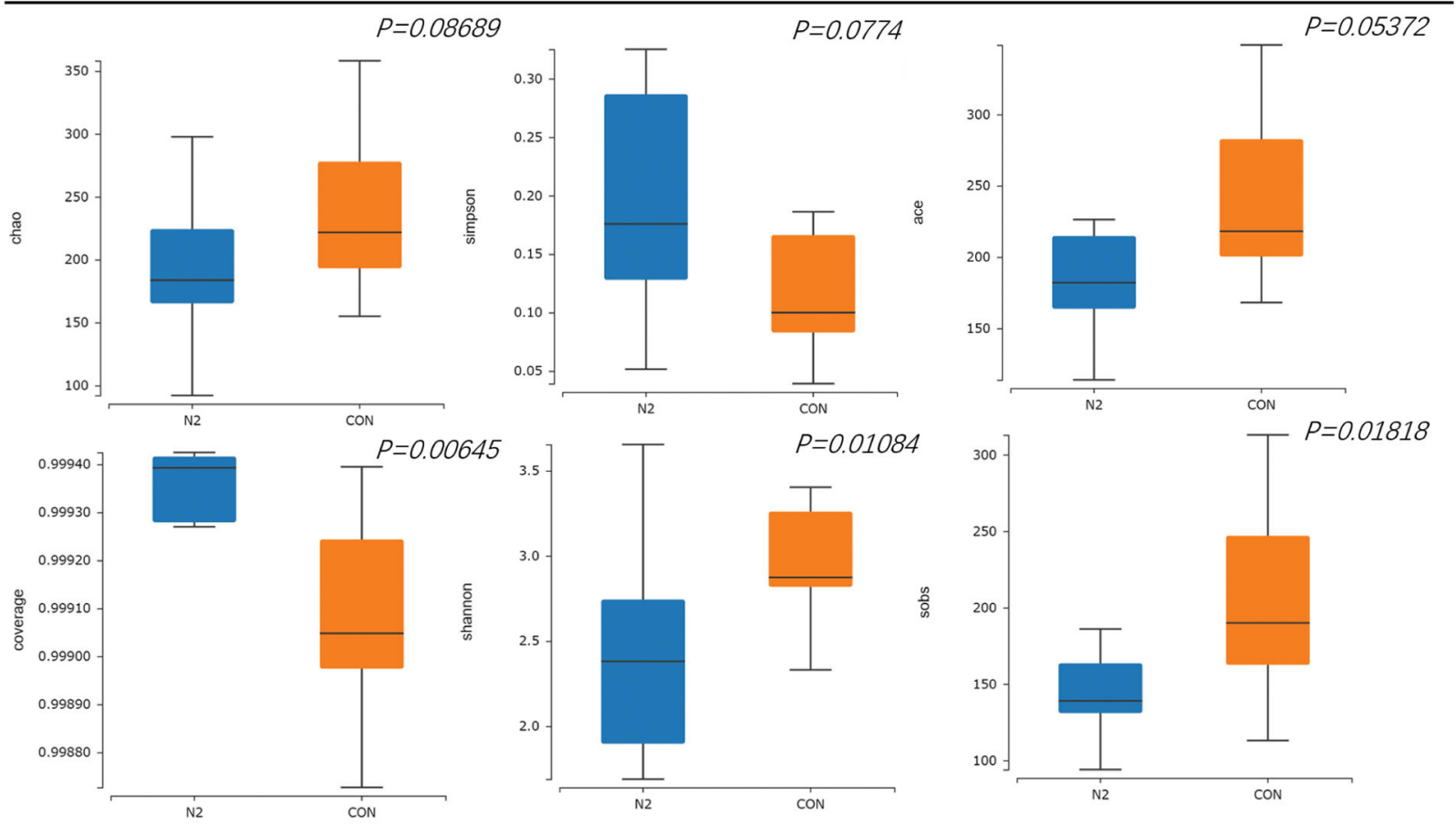

B

\section{C}
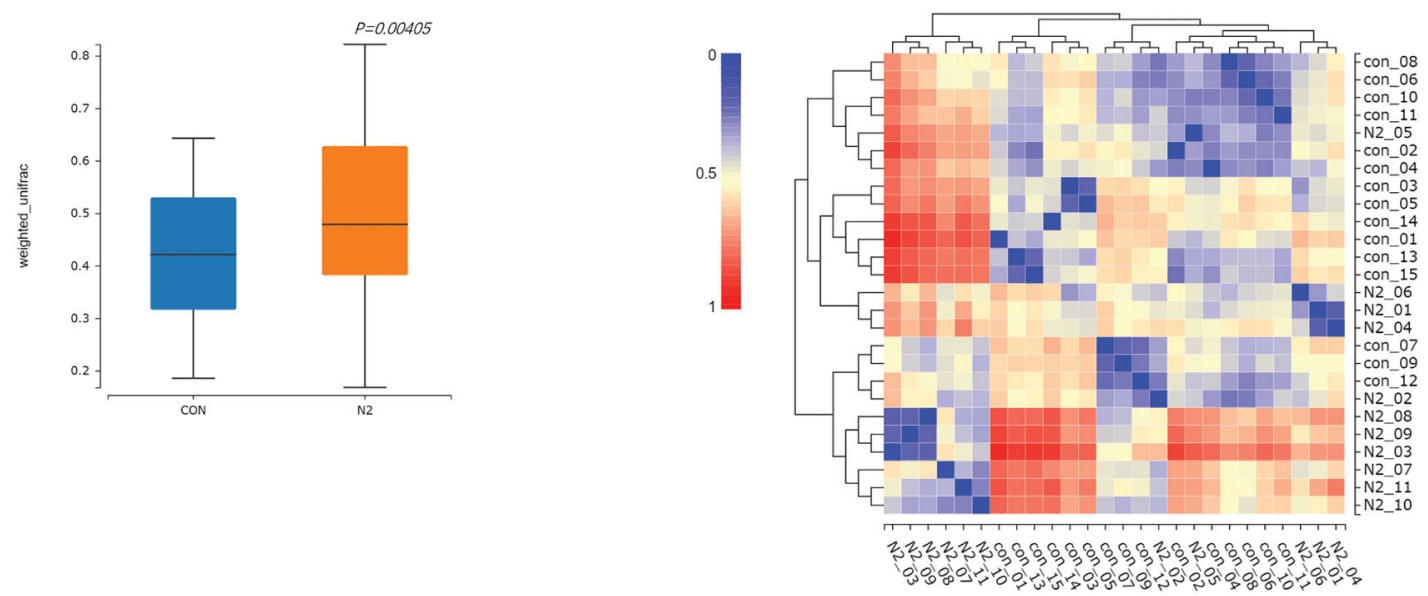

FIGURE 2 | Comparison of $\alpha$-diversity and $\beta$ diversity in healthy controls (CON group) and patients with HBV infection (N2 group). (A) $\alpha$-diversity and (B) $\beta$-diversity, in box plots; (C) $\beta$-diversity in a heatmap.

(Supplementary Figures 3A-C). The microbiota were not separated in the axes PC1 and PC2 in the PCoA plot (Supplementary Figure 4). The microbiota of the N1 group were concentrated mainly in the phyla Bacteroidetes and Fusobacteria (Figure 4A). There were no significant differences in phyla between the N1 group and the N2 group $(p>0.05)$ (Figure 4B). There were 24 differentially expressed genera in the $\mathrm{N} 1$ group compared with those in the HC group (Supplementary Table 2). The abundance of Gemmiger species was lower in the N2 group than that in the N1 group (Figure 4C). A Venn diagram was used to compare the differentially expressed genera of the $\mathrm{N} 1$ group and the N2 group. An altered and distinct profile of Faecalibacterium, Megamonas, and Gemmiger species was observed in the N1 group and N2 group, whereas an altered profile of Phascolarctobacterium and Clostridium_XIVA species occurred only in the N2 group (Supplementary Figure 5A). These results indicated that HBV infection affected the bacterial characteristics of patients with coexistent NAFLD and T2DM. 
A

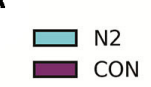

B
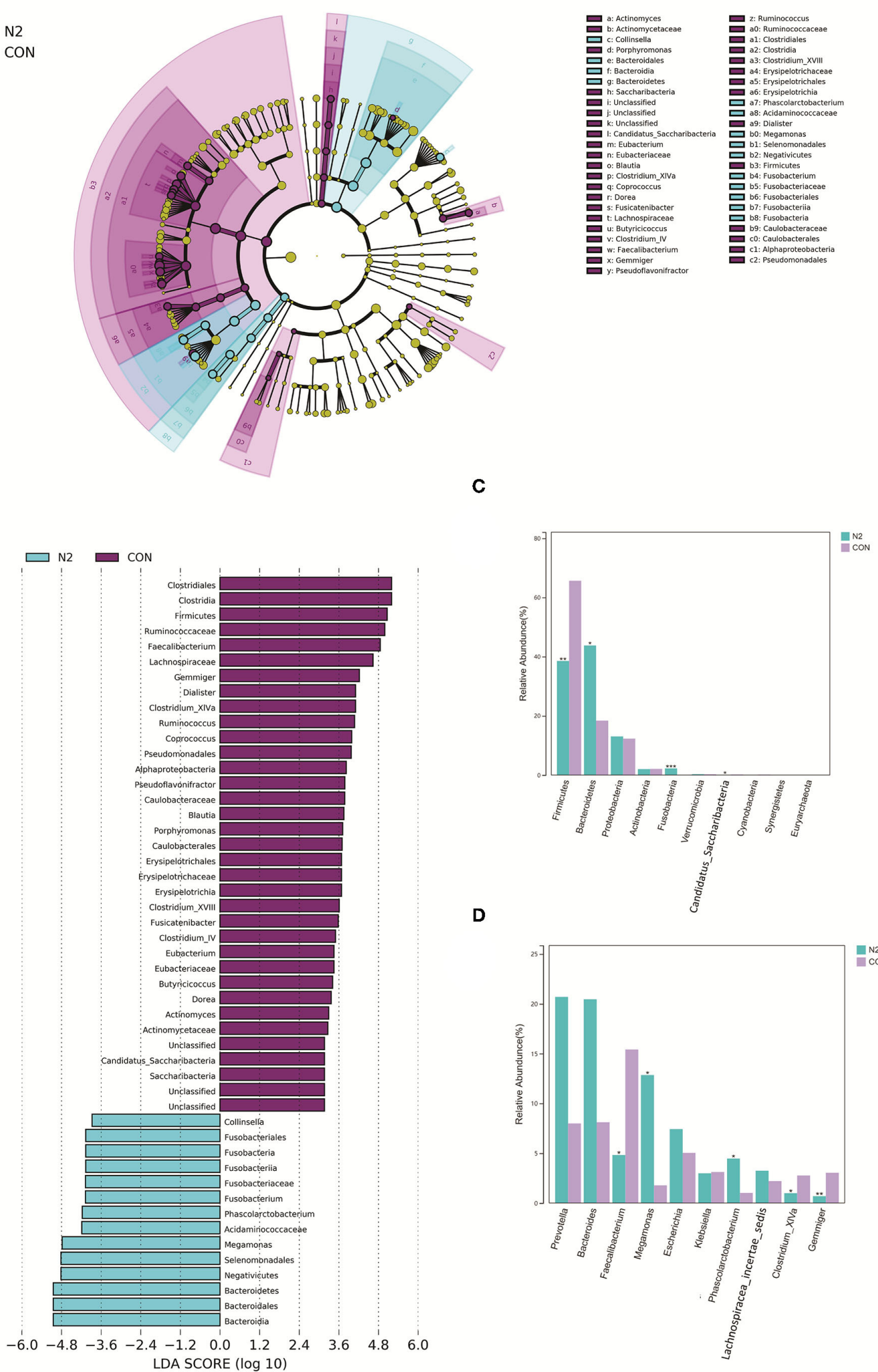

D

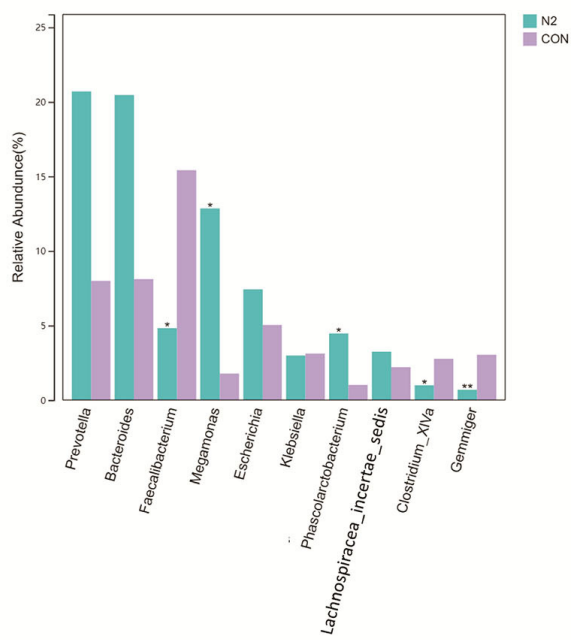

FIGURE 3 | Bacterial composition in healthy controls (CON group) and patients with HBV infection (N2 group). (A) LEfSe cluster diagram of the N2 group. (B) LEfSe LDA of N2 and CON group. (C) Comparison of phyla taxa. (D) Comparison of genera taxa. 
A

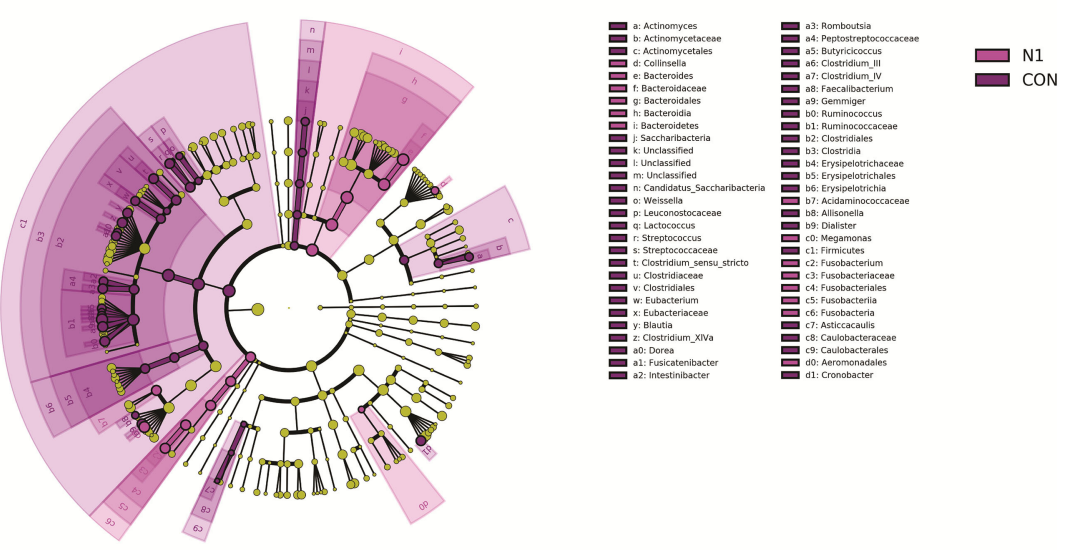

B
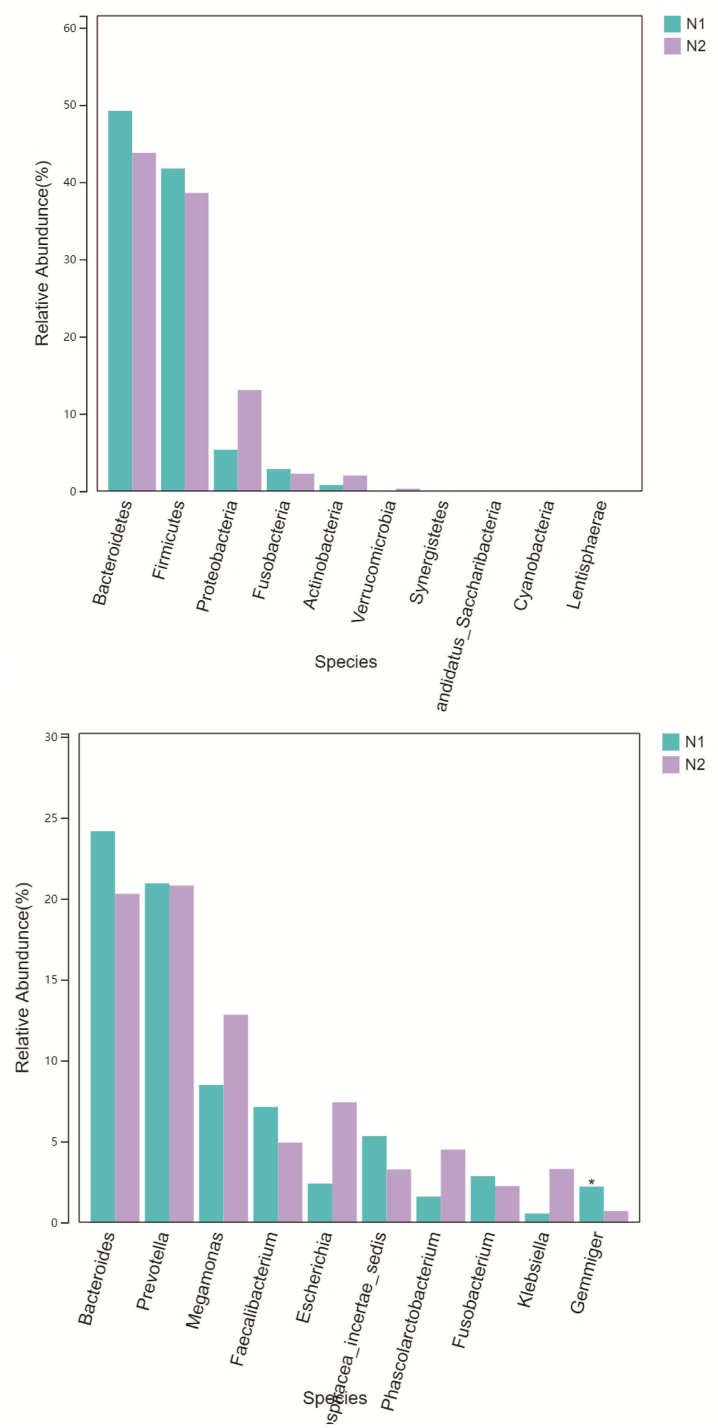

FIGURE 4 | Bacterial composition in patients with HBV infection (N2 group) and patients without HBV infection (N1 group). (A) LEfSe cluster diagram of N1. (B) Comparison of the phyla taxa. (C) Comparison of the phyla taxa. 
A

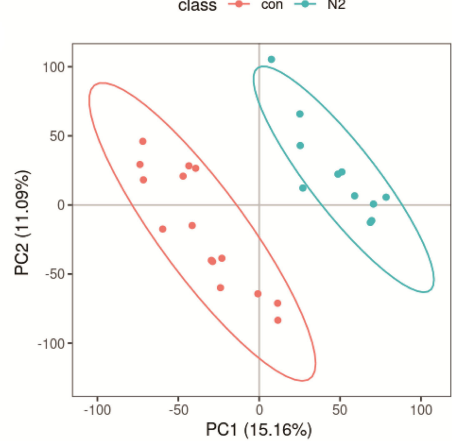

B
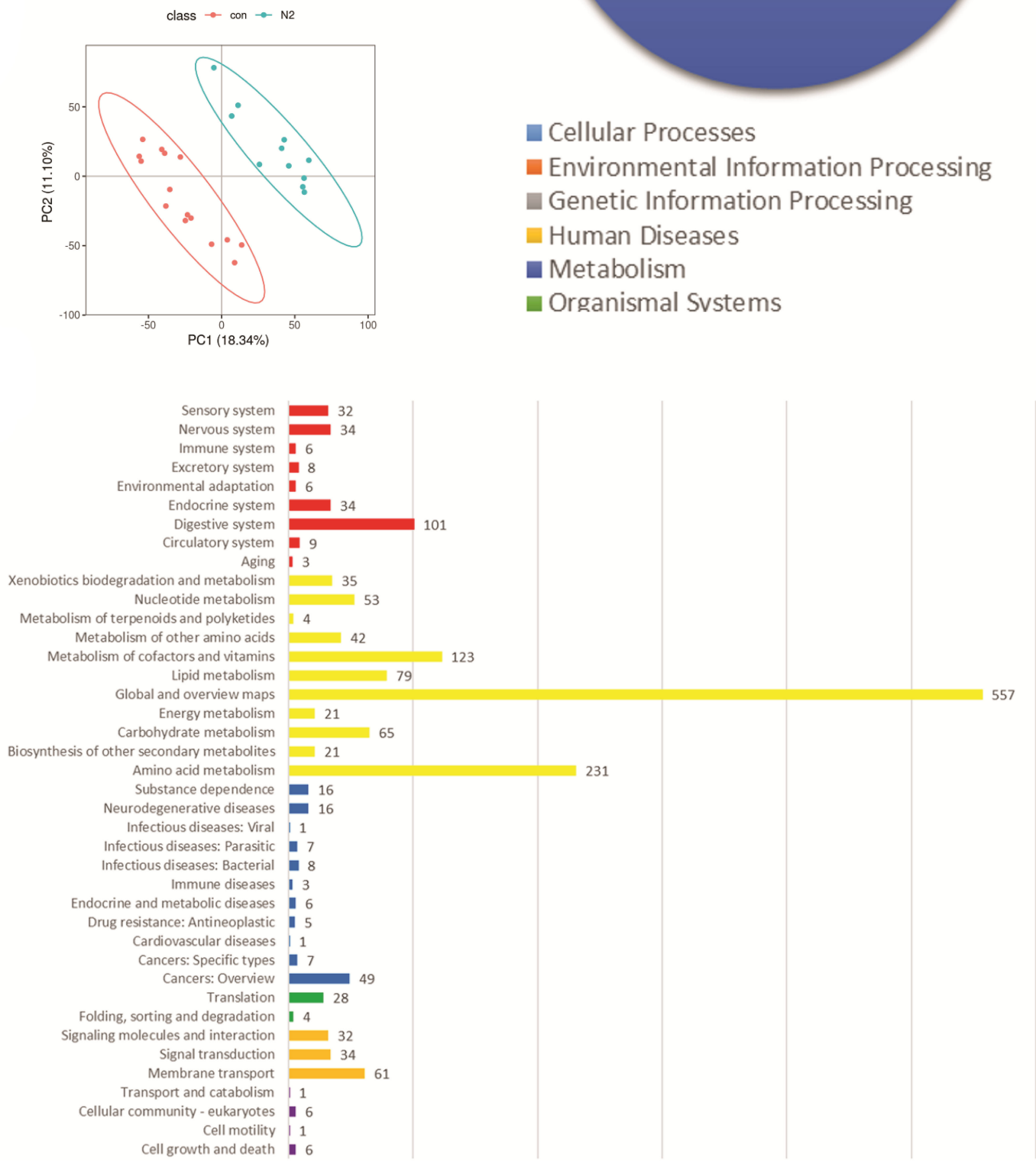

C

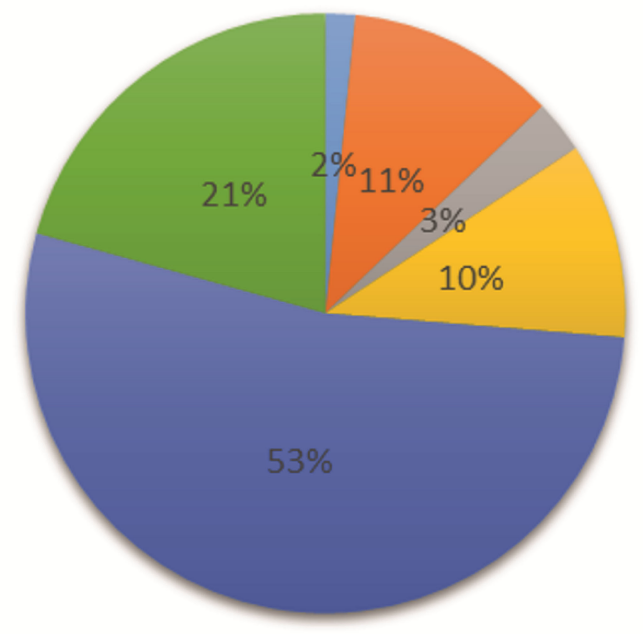

Cellular Processes

- Environmental Information Processing

Genetic Information Processing

- Human Diseases

Metabolism

- Organismal Svstems

\section{D \\ (1)}


A

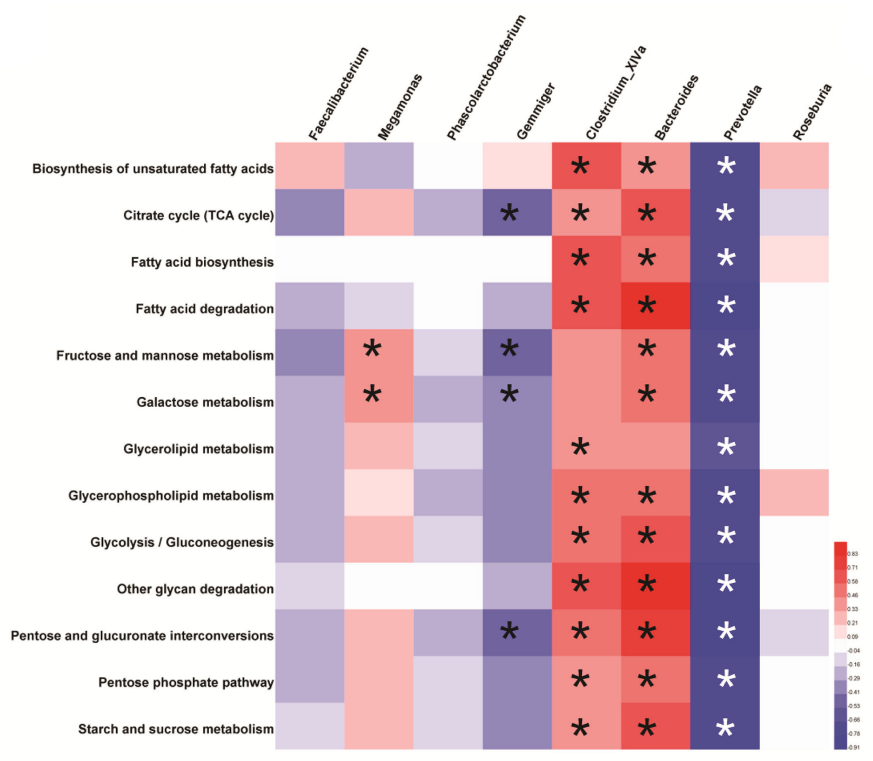

C

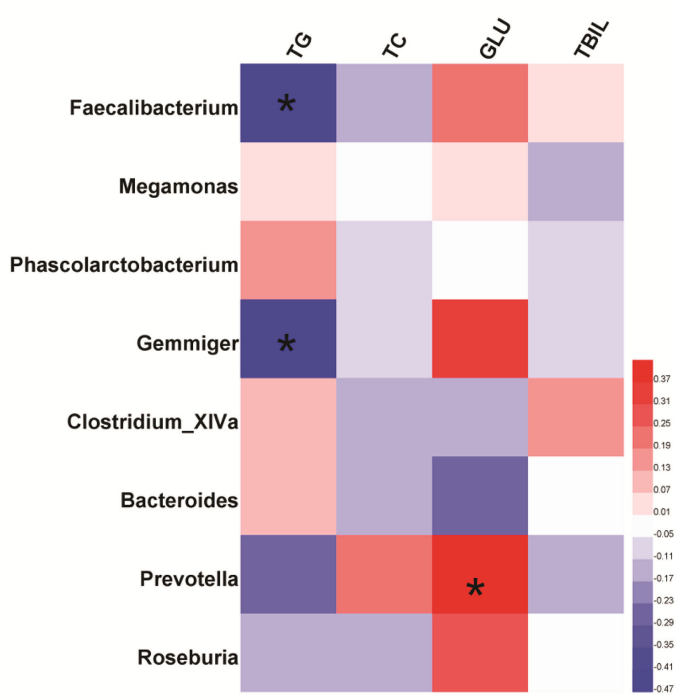

B

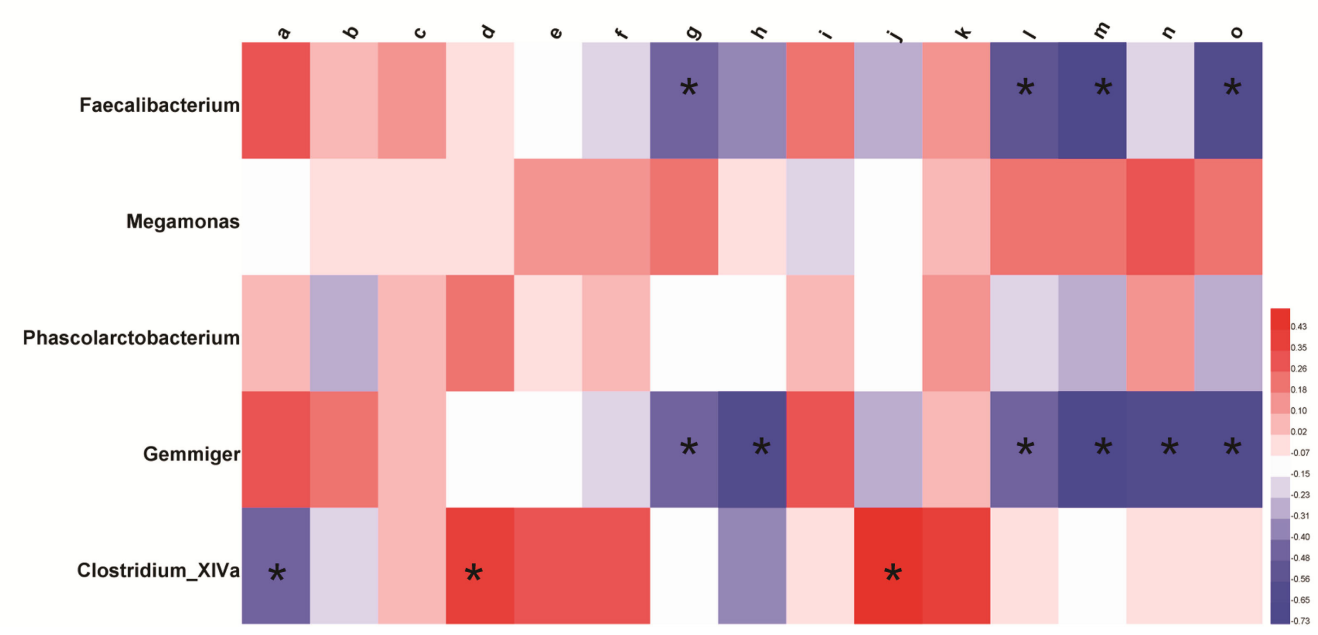

FIGURE 6 | Association between the distinct gut-microbiome profile and metabolism. (A) Featured bacterial genera and pathways related to glucose metabolism and lipid metabolism. The predicted pathway was screened based on the Wilcoxon test $\left(p<0.05\right.$ and $\left.\left|\log _{2} F C\right|>1\right)$. (B) Association between the distinct gut-microbiome profile and differential metabolites related to glucose metabolism and lipid metabolism. Here, a represents $\mathrm{N}$-acetylmuramic acid; $\mathrm{b}$ represents

4-hydroxybenzaldehyde; c represents Cis, cis-muconic acid; d represents 15(s)-hpete; e represents 13(s)-hotre; frepresents 13(s)-hpotre; g represents

Sn-3-o-(geranylgeranyl)glycerol 1-phosphate; $\mathrm{h}$ represents $(3 \alpha, 7 \alpha, 12 \alpha)$-3,7,12,26-tetrahydroxycholestan-27-yl hydrogen sulfate; i represents paromomycin; j

represents L-kynurenine; $\mathrm{k}$ represents 9-oxo-ode; I represents palmitoylcarnitine; $\mathrm{m}$ represents estrone; $n$ represents desoxycortone; o represents cholate. (C)

Featured bacterial genera and clinical manifestations related to glucose metabolism and lipid metabolism. ${ }^{*} p<0.05$. Red represents a positive correlation and, the

darker the color, the more significant is the correlation; blue represents a negative correlation and, the darker the color, the stronger is the correlation.

The projects in the GMrepo database also suggested decreased abundance of Faecalibacterium species and increased abundance of Megamonas species in HBV infection, NAFLD, and T2DM projects. Expression of Gemmiger species was higher in the T2DM project. Alteration of Clostridium species was more pronounced in $\mathrm{HBV}$-infection projects (Supplementary Figure 5B). These projects in datasets also supported the view that HBV infection affected the bacterial characteristics of patients with coexistent NAFLD and T2DM.

\section{Metabolic Characteristics of Patients With Coexistent HBV Infection, NAFLD, and T2DM}

Liquid chromatography-tandem mass spectrometry was used to evaluate metabolic profiles. PLS-DA showed apparent separation between the $\mathrm{N} 2$ group and $\mathrm{HC}$ group in electrospray ionization (ESI) + and ESI- modes (Figures 5A,B). Heatmaps also revealed apparent separation between the $\mathrm{N} 2$ group and $\mathrm{HC}$ group in ESI+ and ESI- modes (Supplementary Figures 6, 7). A 
total of 768 and 1,743 features in ESI- and ESI+ modes were altered significantly between the $\mathrm{N} 2$ group and $\mathrm{HC}$ group according to the selection criteria of variable importance in projection (VIP) $>1,\left|\log _{2} \mathrm{FC}\right|>1.2$, and $p<0.05$. Most of the metabolites belonged to metabolism and organismal systems (Figure 5C, Supplementary Table 3). Enrichment in glucose and lipid metabolic pathways was documented (Figure 5D). The metabolites in carbohydrates, carbonyl compounds, fatty acyls and derivatives, and sterol lipid family showed significant differential expression in the $\mathrm{N} 2$ group, including decreased expression of $\mathrm{N}$-acetylmuramic acid, 4-hydroxybenzaldehyde, Cis, cis-muconic acid, 15(s)-hpete, 13(s)-hotre, 13(s)-hpotre, paromomycin, L-kynurenine, and 9-oxo-ode, and increased expression of Sn-3-o-(geranylgeranyl)glycerol 1-phosphate, $(3 \alpha, 7 \alpha, 12 \alpha)-3,7,12,26$-tetrahydroxycholestan-27-yl hydrogen sulfate, palmitoylcarnitine, estrone, desoxycortone, and cholate (Supplementary Tables 3-6). Palmitoylcarnitine is the metabolite of a fatty-acid metabolic pathway. An increase in the expression of palmitoylcarnitine indicated an increased level of fatty acids (Supplementary Figures 8, 9). The 15(s)-hpete is a metabolite of the arachidonic-acid metabolic pathway. A decrease in the expression of 15(s)-hpete indicated a reduced level of arachidonic acid (Supplementary Figure 10). Sn3-o-(geranylgeranyl)glycerol 1-phosphate is a metabolite of the glycerophospholipid metabolic pathway. An increase in the expression of Sn-3-o-(geranylgeranyl)glycerol 1phosphate indicated an increased level of glycerophospholipids (Supplementary Figure 11).

\section{HBV Infection Affects the Metabolic Characteristics of Patients With Coexistent NAFLD and T2DM}

The PLS-DA showed an apparent separation between the N2 group and N1 group in ESI+ and ESI- modes (Supplementary Figures 12A,B). Analyses of enrichment of pathways using the KEGG database also showed a significant difference in the metabolic pathways between the two groups in ESI+ and ESI- modes (Supplementary Figures 12C,D). A total of 391 and 830 features in ESI- and ESI+ modes were altered significantly between the N2 group and N1 group (Supplementary Tables 7, 8). Compared with the N1 group, expression of Sn-3-o-(geranylgeranyl)glycerol 1-phosphate and $(3 \alpha, 7 \alpha, 12 \alpha)-3,7,12,26$-tetrahydroxycholestan-27-yl hydrogen sulfate was upregulated in the $\mathrm{N} 2$ group according to $\mathrm{VIP}>1$, $\left|\log _{2} \mathrm{FC}\right|>1.2$, and $p<0.05$ (Supplementary Table 9). These results indicated that $\mathrm{HBV}$ infection affected the metabolic characteristics of patients with coexistent NAFLD and T2DM.

\section{Association Between the Distinct GM Profile and Metabolites in Patients With Coexistent HBV Infection, NAFLD, and T2DM}

Enrichment of functional pathways was predicted by Picrust. Glucose- and lipid metabolism-related pathways in KEGG level three were compared with the distinct GM profile through the Spearman correlation analysis. We noted integration between the distinct GM profile and lipid metabolic pathways and the glucose metabolic pathways. Megamonas species were significantly positively correlated with fructose and mannose metabolic pathways and galactose metabolism $(p<0.05)$. Gemmiger species were significantly negatively correlated with the tricarboxylic acid (TCA) cycle, pentose and glucuronate interconversions, fructose and mannose metabolic pathways, and galactose metabolism $(p<0.05)$. Clostridium_XIVA species were significantly positively correlated with several pathways of glucose and lipid metabolism, including the TCA cycle (Figure 6A and Supplementary Table 10).

The Spearman correlation analysis showed integration between the distinct GM profile and metabolites in lipid and glucose metabolic pathways. Clostridium_XlVa species were positively correlated with 15(s)-hpete and L-kynurenine, and negatively correlated with $\mathrm{N}$-acetylmuramic acid. Sn-3o-(geranylgeranyl)glycerol 1-phosphate, palmitoylcarnitine, estrone, and cholate were negatively correlated with Faecalibacterium and Gemmiger species. $(3 \alpha, 7 \alpha, 12 \alpha)-3,7,12,26-$ tetrahydroxycholestan-27-yl hydrogen sulfate and desoxycortone were negatively correlated with Gemmiger species (Figure 6B and Supplementary Table 11).

The distinct GM profile was significantly correlated with levels of TG, TC, and glucose. An increased abundance of Faecalibacterium and Gemmiger species was negatively correlated with the TG level $(p<0.05)$. Prevotella species, whose abundance was increased indirectly through Megamonas species, were positively correlated with the glucose level (Figure 6C and Supplementary Table 12).

\section{DISCUSSION}

Hepatitis B virus has been reported to affect the bacterial characteristics of the host. The composition of the microbiota in patients with $\mathrm{HBV}$ infection may not be identical to that of people not infected with the HBV. We focused, for the first time, on the composition and functional features of the GM and chronic HBV infection in patients with coexistent NAFLD and T2DM. In this way, we aimed to provide new insights into host-GM interactions in this population.

The diversity and richness of bacteria in the gut are important for maintaining intestinal homeostasis, the intestinal mucosal barrier, the immune system, and metabolism of the host. Reduced diversity is a characteristic of microbiota dysbiosis (21-23). In this work, $\alpha$-diversity and $\beta$-diversity were reduced significantly in patients with coexistent HBV infection, NAFLD, and T2DM. Typically, pathobionts (organisms that can cause harm under certain circumstances) are maintained at low levels within the healthy gut, but changes in the composition may lead to an increased number of pathogenic bacteria $(24,25)$. Some studies have suggested that the increased abundance of bacteria of the phylum Firmicutes, a decreased abundance of bacteria of the phylum Bacteroidetes, and an increased Firmicutes/Bacteroidetes ratio are associated with NAFLD prognosis $(26,27)$. However, some researchers have suggested otherwise. They have found that Firmicutes continue to consist of seven probiotic classes 
[including many types of lactic-acid probiotics (28)], whereas bacteria belonging to Bacteroidetes and Fusobacteria genera are increased in many chronic diseases $(16,17)$. Therefore, compositional changes at the genus level are important (27). We found that the bacterial composition of patients with coexistent HBV infection, NAFLD, and T2DM was concentrated in the Bacteroidetes and Fusobacteria phylum, whereas the microbiota of health were concentrated in Firmicutes phylum. Twenty-one genera showed differential expression in those patients. Faecalibacterium, Gemmiger, and Clostridium_XIVA genera showed decreased abundance and Megamonas and Phascolarctobacterium showed an increased abundance in a distinct GM profile. Zeng et al. also suggested that $\mathrm{HBV}$ infection led to a decreased abundance of bacteria of the genera Faecalibacterium and Clostridium (16).

Studies have found an association between HBV infection and reduction in bacterial diversity (16). Researchers have revealed an association between HBV infection and altered bacterial composition in chronic liver diseases $(16,17)$. Zhu and colleagues found that the bacterial diversity and total count of OTUs were changed significantly in mice infected with the HBV (29). In this study, compared with patients with coexistent HBV infection, NAFLD, and T2DM, there was no significant difference in the bacterial diversity in patients not infected with the HBV. In the latter, the bacterial composition was also focused on the Bacteroidetes and Fusobacterium phylum. At the genus level, altered abundance of Phascolarctobacterium and Clostridium_XIVA occurred only in patients infected with the HBV. Furthermore, a decreased abundance of Gemmiger species was more obvious in patients with $\mathrm{HBV}$ infection than in those without HBV infection. These data indicated that HBV infection affected the bacterial characteristics of patients with coexistent NAFLD and T2DM. Wu et al. (8) established the GMrepo database, which enables the exploration of the bacterial characteristics of common diseases at the genus level. Projects in that database also suggested that HBV infection affected the distinct GM profile.

Researchers have referred to the HBV as a "metabolovirus." A recent study suggested that viruses may "reprogram" the host metabolism toward a more lipogenic and adipogenic status (30). HBV X upregulates the expression of fatty acid-binding protein and plays a central part in glucose and de novo lipid synthesis (31). The HBV is thought to be a superposition of hepatic steatosis, glucose metabolism, and hepatic lipidaccumulation pathways $(32,33)$. Therefore, the metabolites and metabolic characteristics of patients with coexistent HBV infection, NAFLD, and T2DM must be explored. Through metabolomics analysis, we found that the metabolites in carbohydrates, carbonyl compounds, fatty acyls and derivatives, and sterol lipids family were altered significantly in patients with coexistent HBV infection, NAFLD, and T2DM. Compared with people not infected with the $\mathrm{HBV}$, those with $\mathrm{HBV}$ infection had a higher level of metabolites related to metabolic pathways, such as Sn-3-o-(geranylgeranyl)glycerol 1-phosphate.

We also revealed an integrated "cross-omics" analysis to better understand the link between the GM and metabolome in patients with coexistent HBV infection, NAFLD, and T2DM. Studies have suggested that bacteria of Gemmiger and Faecalibacterium genera affect metabolites significantly and are involved in the metabolism of fatty acids and glycerophospholipids (34). In this study, Faecalibacterium, Megamonas, and Phascolarctobacterium species facilitated carbohydrate-fermentation pathways, including the TCA cycle. Gemmiger species were negatively related to glucose metabolism, including the TCA cycle and galactose metabolism. Short-chain fatty acids (SCFAs) reduce lipid accumulation and insulin resistance (35). SCFAs-producing microbiotas are involved in gluconeogenesis, reduce the TG level, and promote energy storage (36). Studies have suggested that the SCFAs-producing microbiotas are reduced in NAFLD (37), T2DM (38), and HBV infection (39). Similarly, the distinct GM profile in patients with coexistent HBV infection, NAFLD, and T2DM in our study, including Faecalibacterium, Megamonas, and Phascolarctobacterium genera involved SCFA production, whereas Gemmiger species did not (40-44).

Studies have suggested that the expression of palmitoylcarnitine (product of fatty-acid metabolism) was upregulated along with an increased abundance of Faecalibacterium species (45). We found that palmitoylcarnitine in the fatty-acid metabolic pathway and Sn-3-o-(geranylgeranyl)glycerol 1-phosphate in the glycerophospholipid metabolic pathway were negatively correlated to Faecalibacterium and Gemmiger genera. Studies have suggested that the N-terminal domain of Clostridium perfringens alpha-toxin shows sequence and predicted structural homologies with the $\mathrm{N}$-terminal region of arachidonate 5-lipoxygenase (46). We found that 15(s)-hpete in the arachidonic-acid metabolic pathway was positively correlated with Clostridium_XIVA species.

Patients with NAFLD with HBV infection have been shown to have significantly higher serum levels of fasting glucose, TG, and TC than those NAFLD patients not suffering from HBV infection (47). CHB is followed by increased levels of fatty acids, hepatic glucose production, and impaired glucose tolerance $(48,49)$. The clinical indicators in the present study also showed a negative relationship between the TG level and Faecalibacterium and Gemmiger genera. The abundance of Prevotella species was increased indirectly through Megamonas species (Supplementary Figure 13 and Supplementary Table 13) and was positively correlated with the glucose level.

The main limitation of our study was the relatively small cohort. However, based on projects in the GMrepo database and previous studies, we found a strong relationship between the altered bacterial composition and HBV infection in patients with coexistent NAFLD and T2DM.

\section{CONCLUSIONS}

We revealed an association between altered bacterial characteristics and $\mathrm{HBV}$ infection in patients with coexistent NAFLD and T2DM. The distinct GM profile may have a functional role in glucose metabolism and lipid metabolism. Our study provides a basis for GM regulation as a potential therapeutic target for patients with coexistent HBV infection, NAFLD, and T2DM living in southern China. 


\section{DATA AVAILABILITY STATEMENT}

The datasets presented in this study can be found in online repositories. The names of the repository/repositories and accession number(s) can be found at: https://www.ncbi.nlm.nih. gov/, BioProject ID: PRJNA780085.

\section{ETHICS STATEMENT}

The studies involving human participants were reviewed and approved by Nanfang Hospital, Southern Medical University, Guangzhou, China (Approval No. NFEC-2018-023; ClinicalTrials.gov ID. NCT03525769). The patients/participants provided their written informed consent to participate in this study.

\section{AUTHOR CONTRIBUTIONS}

WH: performed the statistical analysis. $\mathrm{CH}$ and YJ: carried out the study and collected stools specimens. LZ: assistance for data acquisition and analysis. JC and JH: carried out literature research and manuscript editing. All authors contributed to the article and approved the submitted version.

\section{FUNDING}

This article was supported by (1) Sanming Project of Medicine in Shenzhen (No. SZSM 201911001); (2) Research Foundation of Shenzhen Hospital of Southern Medical University (PY2021YM04); (3) National Natural Science Foundation of China (Grant No. 82000544); (4) Beijing iGandan Foundation (RGGJJ-2021-029); (5) Beijing Municipal Administration of Hospitals Incubating Program (Code: PX2019062); and (6) China Postdoctoral Science Foundation (2021M701612).

\section{ACKNOWLEDGMENTS}

We thank all the participants and volunteers for sample collection and face-to-face interviews.

\section{REFERENCES}

1. Tsai HJ, Tsai YC, Hung WW, Hung WC, Chang CC, Dai CY. Gut microbiota and non-alcoholic fatty liver disease severity in type 2 diabetes patients. J Pers Med. (2021) 11:238. doi: 10.3390/jpm11030238

2. Thibaut R, Gage MC, Pineda-Torra I, Chabrier G, Venteclef N, Alzaid F, Liver macrophages and inflammation in physiology and physiopathology of non-alcoholic fatty liver disease. FEBS J. (2021). doi: 10.1111/febs.15877

3. Mansour A, Mohajeri-Tehrani MR, Samadi M, Qorbani M, Merat S, Adibi H. Effects of supplementation with main coffee components including caffeine and/or chlorogenic acid on hepatic, metabolic, and inflammatory indices in patients with non-alcoholic fatty liver disease and type 2 diabetes: a randomized, double-blind, placebo-controlled, clinical trial. Nutr J. (2021) 20:35. doi: 10.1186/s12937-021-00694-5

4. Bessone F, Razori MV, Roma MG. Molecular pathways of nonalcoholic fatty liver disease development and progression. Cell Mol Life Sci. (2019) 76:99128. doi: 10.1007/s00018-018-2947-0

\section{SUPPLEMENTARY MATERIAL}

The Supplementary Material for this article can be found online at: https://www.frontiersin.org/articles/10.3389/fmed. 2021.805029/full\#supplementary-material

Supplementary Figure 1 | Rarefaction curves of all samples. (A) Shannon Index. (B) Observed species.

Supplementary Figure 2| PCoA revealing the bacterial communities between the $\mathrm{N} 2$ group and CON group.

Supplementary Figure $3 \mathbf{~ | ~ C o m p a r i s o n ~ o f ~} \alpha$-diversity and $\beta$-diversity in patients with HBV infection (N2 group) and patients without HBV infection (N1 group). (A) $\alpha$-diversity and (B) $\beta$-diversity in box plots. (C) $\beta$-diversity in a heatmap; *Diversity coefficient $>0.6$.

Supplementary Figure 4 | PCoA revealing the bacterial communities between the N1 group and N2 group.

Supplementary Figure 5 | (A) Venn diagram of distinguished expressed genera in patients with HBV infection (N2 group) and those without HBV infection (N1 group). (B) Abundance of the distinct gut-microbiome profile in the GMrepo database.

Supplementary Figure 6 | Heatmap of differential metabolites in ESI+ mode. Red represents a positive relationship; green represents a negative relationship. $\mathrm{ESI}$, electrospray ionization.

Supplementary Figure 7 | Heatmap of differential metabolites in ESI-mode. Red represents a positive relationship; green represents a negative relationship. ESI, electrospray ionization.

Supplementary Figure 8| Palmitoylcarnitine in fatty acid-degradation pathway.

Supplementary Figure 9 | Palmitoylcarnitine in fatty acid-degradation metabolic pathway.

Supplementary Figure 10 | 15(s)-hpete in an arachidonic-acid metabolic pathway.

Supplementary Figure 11 | Sn-3-o-(geranylgeranyl)glycerol 1-phosphate in a glycerophospholipid metabolic pathway.

Supplementary Figure 12 | Fecal metabolic profiles in the N2 group and N1 group. (A) PLS-DA of the two groups in ESI+ mode. (B) PLS-DA of the two groups in ESI-mode. (C) Enriched pathways of altered metabolic features in ESI+ mode according to the KEGG database. (D) Enriched pathways of altered metabolic features in ESI- mode according to the KEGG database.

Supplementary Figure 13 | The complicated network pattern between the distinct gut-microbiome profile. *Correlation coefficient $>0.5$ or $<-0.5$.

5. Cai J, Zhang XJ, Ji YX, Zhang P, She ZG, Li H. Nonalcoholic fatty liver disease pandemic fuels the upsurge in cardiovascular diseases. Circ Res. (2020) 126:679-704. doi: 10.1161/CIRCRESAHA.119.316337

6. Kim H, Worsley O, Yang E, Purbojati RW, Liang AL, Tan W. Persistent changes in liver methylation and microbiome composition following reversal of diet-induced non-alcoholic-fatty liver disease. Cell Mol Life Sci. (2019) 76:4341-54. doi: 10.1007/s00018-019-03114-4

7. Uittenbogaart M, Leclercq WK, Bonouvrie D, Romeijn MM, Luijten AA, Olde Damink SW. Diet-induced alteration of microbiota and development of obesity, nonalcoholic fatty liver disease, and diabetes: study protocol of a prospective study. JMIR Res Protoc. (2019) 8:e11553. doi: 10.2196/11553

8. Wu S, Sun C, Li Y, Wang T, Jia L, Lai S. GMrepo: a database of curated and consistently annotated human gut metagenomes. Nucleic Acids Res. (2020) 48:D545-D53. doi: 10.1093/nar/gkz764

9. Wu H, Tremaroli V, Schmidt C, Lundqvist A, Olsson LM, Krämer M. The gut microbiota in prediabetes and diabetes: a population-based cross-sectional study. Cell Metab. (2020) 32:379-90.e3. doi: 10.1016/j.cmet.2020.06.011 
10. Aron-Wisnewsky J, Vigliotti C, Witjes J, Le P, Holleboom AG, Verheij J. Gut microbiota and human NAFLD: disentangling microbial signatures from metabolic disorders. Nat Rev Gastroenterol Hepatol. (2020) 17:27997. doi: 10.1038/s41575-020-0269-9

11. Chen Y, Feng R, Yang X, Dai J, Huang M, Ji X. Yogurt improves insulin resistance and liver fat in obese women with nonalcoholic fatty liver disease and metabolic syndrome: a randomized controlled trial. Am J Clin Nutr. (2019) 109:1611-9. doi: 10.1093/ajcn/nqy358

12. Wu H, Esteve E, Tremaroli V, Khan MT, Caesar R, Mannerås-Holm L. Metformin alters the gut microbiome of individuals with treatment-naive type 2 diabetes, contributing to the therapeutic effects of the drug. Nat Med. (2017) 23:850-8. doi: 10.1038/nm.4345

13. Sharif A, Abbas Z, Ahmed S, Ali Samjo S, Baqai K. Effect of non-alcoholic fatty liver disease on transaminase levels and transient elastography in patients with chronic hepatitis B. Cureus. (2019) 11:e5995. doi: 10.7759/cureus.5995

14. Kim M, Lee Y, Yoon JS, Lee M, Kye SS, Kim SW. The FIB-4 index is a useful predictor for the development of hepatocellular carcinoma in patients with coexisting nonalcoholic fatty liver disease and chronic hepatitis B. Cancers. (2021) 13:2301. doi: 10.3390/cancers13102301

15. Suliman I, Abdelgelil N, Kassamali F, Hassanein TI. The effects of hepatic steatosis on the natural history of HBV infection. Clin Liver Dis. (2019) 23:433-50. doi: 10.1016/j.cld.2019.05.001

16. Zeng Y, Chen S, Fu Y, Wu W, Chen T, Chen J. Gut microbiota dysbiosis in patients with hepatitis B virus-induced chronic liver disease covering chronic hepatitis, liver cirrhosis and hepatocellular carcinoma. J Viral Hepat. (2020) 27:143-55. doi: 10.1111/jvh.13216

17. Wang J, Wang Y, Zhang X, Liu J, Zhang Q, Zhao Y. Gut microbial dysbiosis is associated with altered hepatic functions and serum metabolites in chronic hepatitis B patients. Front Microbiol. (2017) 8:2222. doi: $10.3389 /$ fmicb. 2017.02222

18. Eddowes PJ, Sasso M, Allison M, Tsochatzis E, Anstee QM, Sheridan D. Accuracy of FibroScan controlled attenuation parameter and liver stiffness measurement in assessing steatosis and fibrosis in patients with nonalcoholic fatty liver disease. Gastroenterology. (2019) 156:171730. doi: 10.1053/j.gastro.2019.01.042

19. Xiao G, Zhu S, Xiao X, Yan L, Yang J, Wu G. Comparison of laboratory tests, ultrasound, or magnetic resonance elastography to detect fibrosis in patients with nonalcoholic fatty liver disease: a meta-analysis. Hepatology. (2017) 66:1486-501. doi: 10.1002/hep.29302

20. Kwan SY, Jiao J, Joon A, Wei P, Petty LE, Below JE. Gut microbiome features associated with liver fibrosis in Hispanics, a population at high risk for fatty liver disease. Hepatology. (2021). doi: 10.1002/hep.32197

21. Liu Z, Liao W, Zhang Z, Sun R, Luo Y, Chen Q. Metformin affects gut microbiota composition and diversity associated with amelioration of dextran sulfate sodium-induced colitis in mice. Front Pharmacol. (2021) 12:640347. doi: 10.3389/fphar.2021.640347

22. Mihajlovic J, Leutner M, Hausmann B, Kohl G, Schwarz J, Röver H. Combined hormonal contraceptives are associated with minor changes in composition and diversity in gut microbiota of healthy women. Environ Microbiol. (2021) 23:3037-47. doi: 10.1111/1462-2920.15517

23. Rizzetto L, De Filippo C, Cavalieri D. Richness and diversity of mammalian fungal communities shape innate and adaptive immunity in health and disease. Eur J Immunol. (2014) 44:3166-81. doi: 10.1002/eji.201344403

24. Petersen C, Round JL. Defining dysbiosis and its influence on host immunity and disease. Cell Microbiol. (2014) 16:1024-33. doi: 10.1111/cmi.12308

25. Chow J, Mazmanian SK. A pathobiont of the microbiota balances host colonization and intestinal inflammation. Cell Host Microbe. (2010) 7:26576. doi: 10.1016/j.chom.2010.03.004

26. Koliada A, Syzenko G, Moseiko V, Budovska L, Puchkov K, Perederiy V. Association between body mass index and Firmicutes/Bacteroidetes ratio in an adult Ukrainian population. BMC Microbiol. (2017) 17:120. doi: 10.1186/s12866-017-1027-1

27. Magne F, Gotteland M, Gauthier L, Zazueta A, Pesoa S, Navarrete P. The firmicutes/bacteroidetes ratio: a relevant marker of gut dysbiosis in obese patients? Nutrients. (2020) 12:1474. doi: 10.3390/nu120 51474

28. Seong CN, Kang JW, Lee JH, Seo SY, Woo JJ, Park C. Taxonomic hierarchy of the phylum firmicutes and novel firmicutes species originated from various environments in Korea. J Microbiol. (2018) 56:1-10. doi: 10.1007/s12275-018-7318-x

29. Zhu Q, Xia P, Zhou X, Li X, Guo W, Zhu B. Hepatitis B virus infection alters gut microbiota composition in mice. Front Cell Infect Microbiol. (2019) 9:377. doi: $10.3389 /$ fcimb. 2019.00377

30. Tarantino G, Citro V, Cataldi M. Findings from studies are congruent with obesity having a viral origin, but what about obesity-related NAFLD? Viruses. (2021) 13:1285. doi: 10.3390/v13071285

31. Cheng C, Geng F, Cheng X, Guo D. Lipid metabolism reprogramming and its potential targets in cancer. Cancer Commun. (2018) 38:27. doi: 10.1186/s40880-018-0301-4

32. Razi B, Alizadeh S, Omidkhoda A, Imani D, Rezaei R. Association of chronic hepatitis B infection with metabolic syndrome and its components: metaanalysis of observational studies. Diabetes Metab Syndr. (2017) 11 Suppl 2:S939-s47. doi: 10.1016/j.dsx.2017.07.020

33. Bar-Yishay I, Shaul Y, Shlomai A. Hepatocyte metabolic signalling pathways and regulation of hepatitis B virus expression. Liver Int. (2011) 31:28290. doi: 10.1111/j.1478-3231.2010.02423.x

34. Zhang D, Liu H, Wang S, Zhang W, Wang Y. Sex-dependent changes in the microbiota profile, serum metabolism, and hormone levels of growing pigs after dietary supplementation with Lactobacillus. Appl Microbiol Biotechnol. (2021) 105:4775-89. doi: 10.1007/s00253-021-11310-1

35. Zhang F, He F, Guo L, Li L, Zhang B, Yu S. Bioavailability based on the gut microbiota: a new perspective. Microbiol Mol Biol Rev. (2020) 84. doi: 10.1128/MMBR.00072-19

36. Schwimmer JB, Johnson JS, Angeles JE, Behling C, Belt PH, Borecki, I. Microbiome signatures associated with steatohepatitis and moderate to severe fibrosis in children with nonalcoholic fatty liver disease. Gastroenterology. (2019) 157:1109-22. doi: 10.1053/j.gastro.2019.08.040

37. Rau M, Rehman A, Dittrich M, Groen AK, Hermanns HM, Seyfried F. Fecal SCFAs and SCFA-producing bacteria in gut microbiome of human NAFLD as a putative link to systemic T-cell activation and advanced disease. United European Gastroenterol J. (2018) 6:1496-507. doi: 10.1177/2050640618804444

38. Chumburidze-Areshidze N, Kezeli T, Avaliani Z, Mirziashvili M, Avalian $\mathrm{T}$, Gongadze N. The relationship between type-2 diabetes and tuberculosis. Georgian Med News. (2020) 69-74.

39. Yang XA, Lv F, Wang R, Chang Y, Zhao Y, Cui X. Potential role of intestinal microflora in disease progression among patients with different stages of Hepatitis B. Gut Pathog. (2020) 12:50. doi: 10.1186/s13099-020-00391-4

40. Lopez-Siles M, Duncan SH, Garcia-Gil LJ, Martinez-Medina M. Faecalibacterium prausnitzii: from microbiology to diagnostics and prognostics. ISME J. (2017) 11:841-52. doi: 10.1038/ismej.2016.176

41. Morotomi M, Nagai F, Sakon H. Genus megamonas should be placed in the lineage of firmicutes; clostridia; clostridiales; 'acidaminococcaceae'; megamonas. Int J Syst Evol Microbiol. (2007) 57:1673-4. doi: 10.1099/ijs.0.65150-0

42. Rachek S, Nikpoor N, Gómez Del Pulgar EM, Gonzaga A, Sanz Y, Tompkins TA. Complete genome sequence of phascolarctobacterium faecium G 104 isolated from the stools of a healthy lean donor. Microbiol Resour Announc. (2021) 10:e01054-20. doi: 10.1128/MRA.01054-20

43. Monga Kravetz A, Testerman T, Galuppo B, Graf J, Pierpont B, Siebel S. Effect of gut microbiota and PNPLA3 rs738409 variant on nonalcoholic fatty liver disease (NAFLD) in obese youth. J Clin Endocrinol Metab. (2020) 105:dgaa382. doi: 10.1210/clinem/dgaa382

44. Li TT, Liu YY, Wan XZ, Huang ZR, Liu B, Zhao C. Regulatory efficacy of the polyunsaturated fatty acids from microalgae spirulina platensis on lipid metabolism and gut microbiota in high-fat diet rats. Int J Mol Sci. (2018) 19:3075. doi: 10.3390/ijms19103075

45. Meng XL, Li S, Qin CB, Zhu ZX, Hu WP, Yang LP. Intestinal microbiota and lipid metabolism responses in the common carp (Cyprinus carpio L.) following copper exposure. Ecotoxicol Environ Saf. (2018) 160:25764. doi: 10.1016/j.ecoenv.2018.05.050

46. Titball RW, Leslie DL, Harvey S, Kelly D. Hemolytic and sphingomyelinase activities of clostridium perfringens alpha-toxin are dependent on a domain homologous to that of an enzyme from the human arachidonic acid pathway. Infect Immun. (1991) 59:1872-4. doi: 10.1128/iai.59.5.1872-1874.1991

47. Nau AL, Soares JC, Shiozawa MB, Dantas-Corrêa EB, Schiavon Lde L, Narciso-Schiavon JL. Clinical and laboratory characteristics associated with 
dyslipidemia and liver steatosis in chronic HBV carriers. Rev Soc Bras Med Trop. (2014) 47:158-64. doi: 10.1590/0037-8682-0009-2014

48. Musso G, Gambino R, Cassader M. Recent insights into hepatic lipid metabolism in non-alcoholic fatty liver disease (NAFLD). Prog Lipid Res. (2009) 48:1-26. doi: 10.1016/j.plipres.2008.08.001

49. Lamontagne RJ, Casciano JC, Bouchard MJ. A broad investigation of the HBV-mediated changes to primary hepatocyte physiology reveals HBV significantly alters metabolic pathways. Metabolism. (2018) 83:509. doi: 10.1016/j.metabol.2018.01.007

Conflict of Interest: The authors declare that the research was conducted in the absence of any commercial or financial relationships that could be construed as a potential conflict of interest.
Publisher's Note: All claims expressed in this article are solely those of the authors and do not necessarily represent those of their affiliated organizations, or those of the publisher, the editors and the reviewers. Any product that may be evaluated in this article, or claim that may be made by its manufacturer, is not guaranteed or endorsed by the publisher.

Copyright (๑) 2021 Han, Huang, Ji, Zhou, Chen and Hou. This is an open-access article distributed under the terms of the Creative Commons Attribution License (CC $B Y)$. The use, distribution or reproduction in other forums is permitted, provided the original author(s) and the copyright owner(s) are credited and that the original publication in this journal is cited, in accordance with accepted academic practice. No use, distribution or reproduction is permitted which does not comply with these terms. 